\title{
RIESZ BASIS APPROACH TO THE TRACKING CONTROL OF A FLEXIBLE BEAM WITH A TIP RIGID BODY WITHOUT DISSIPATIVITY
}

\author{
BAO-ZHU GUO* \\ Institute of Systems Science, Academy of Mathematics and \\ System Sciences, Academia Sinica, Beijing 100080, China
}

(Received 27 August 2001; Revised 14 February 2002; In final form 4 April 2002)

A linear feedback control is designed regardless of dissipativity of the system for the stabilization of a flexible beam with a tip rigid body. The Riesz basis approach is adopted in the investigation. It is shown that the closed loop system is a Riesz spectral system and as consequences, the exponential stability, the observability and the controllability of the system are concluded. Finally, some numerical results are also presented.

Keywords: Riesz basis; Spectrum-determined growth condition; Stability;

Controllability; Observability

\section{INTRODUCTION}

In this article, we consider a flexible beam rotated by a motor in a horizontal plane at one end and a tip body rigidly attached at the free end. This model fits a large class of real applications such as links of robot systems and space-shuttle arms in which high speed manipulation and long and slender geometrical dimensions are the major factors causing mechanical vibration. To achieve high speed and precision end point positioning of the flexible beam, the boundary control is one of the major strategies in production and space applications.

\footnotetext{
*E-mail: bzguo@iss03.iss.ac.cn
} 
Let $\ell$ be the length of the beam, $\rho$ the uniform mass density per unit length, $E I$ the uniform flexural rigidity, $\tilde{M}$ the mass of the tip body attached, $\tilde{I}_{m}$ the moment of inertia of the motor and $\tilde{J}$ the moment of inertia associated with the tip body. Suppose that the terminal state trajectory is $x \vartheta_{d}(t)$ at position $x$ and time $t$, where $\vartheta_{d}^{\prime \prime}(t)=0$, i.e., the tracked state would be uniform motion or fixed in some direction of the flexible beam. Let the difference displacement $\tilde{y}(x, t)=z(x, t)-x \vartheta_{d}(t)$, where $z(x, t)$ is the total transversal displacement at $x$ and $t$. Then $\tilde{y}(x, t)$ satisfies the following Euler-Bernoulli beam equation and the Newton-Euler rigid-body equations [1]:

$$
\left\{\begin{array}{l}
\rho \tilde{y}_{t t}(x, t)+E I \tilde{y}_{x x x x}(x, t)=0, \quad 0<x<\ell, t>0, \\
\tilde{y}(0, t)=0, \\
E I \tilde{y}_{x x}(0, t)-\tilde{I}_{m} \tilde{y}_{x t t}(0, t)+u(t)=0, \\
E I \tilde{y}_{x x x}(\ell, t)-\tilde{M} \tilde{y}_{t t}(\ell, t)=0 \\
E I \tilde{y}_{x x}(\ell, t)+\tilde{J} \tilde{y}_{x t t}(\ell, t)=0
\end{array}\right.
$$

where $u(t)$ is the torque developed by the motor. It was shown in [1] that the following nonlinear feedback control

$$
u(t)=-\alpha \tilde{y}_{x}(0, t)-f\left(\tilde{y}_{x t}(0, t)\right)
$$

can make system (1) asymptotically stable or, exponentially stable when $\tilde{I}_{m}=0$, where $\alpha>0$ is a constant and $f \in C(\mathbb{R})$ is increasing with

$$
f(0)=0 \quad \text { and } \quad s f(s)>0 \text { for } s \neq 0 .
$$

However, when $\tilde{I}_{m} \neq 0$, no result is available to the uniform stabilization of this hybrid system.

A similar problem was considered in [2] for string vibration that describes the vibration of an overhead crane. There are many different models in literature describing the vibration of a flexible beam with a tip rigid body $[3,4,14,15]$, we refer to $[3,4]$ for further descriptions concerning the physical structure of the system. To realize the uniform stabilization, the "high derivative" feedback control is usually required (see e.g. [5,6]), for instance, when $f$ is linear in (2), we shall see in Section 2 of this article that control (2) cannot uniformly stabilize the closed-loop system. However, on the one hand, the design of the "high derivative" feedback controllers in literature are mainly based on principle of "passivity" that makes the closed-loop system be 
dissipative so that the system is at least asymptotically stable by Lyapunov function method [5,6]. In applications, on the other hand, there are many ways of designing controllers that make system practically uniformly stable but there is no dissipativity which usually brings the difficulty of theoretical proof for the uniform stability of the system [7]. In this article, we shall design such a "high derivative" feedback controller for system (1) which can make the closed-loop system exponentially stable although we do not know if it is dissipative. The approach used here is so called Riesz basis approach that is recently used to study the basis generation, exponential stability and distribution of eigenvalues of the Euler-Bernoulli beam equations in a very simple way $[8,16]$. In the next section, an unbounded boundary feedback control is designed and the asymptotic expressions of eigenvalues and eigenfunctions are derived. In Section 3, we show that there is a sequence of generalized eigenfunctions of system (1), which forms a Riesz basis for the state Hilbert space. Section 4 is devoted to the exponential stability of the system. Controllability and observability are obtained in Section 5. Numerical simulation is presented in Section 6 after relating the stability of the system to a finite dimensional eigenvalue problem. Some concluding remarks are made in Section 7.

\section{ASYMPTOTIC EXPRESSION OF EIGENPAIRS}

In order to adopt the Riesz basis approach to study the stability of system (1) as in $[8,16]$, we should first formulate the closed-loop system into a linear evolution equation in an underlying Hilbert state space and then find asymptotic expressions of eigenpairs of the system operator associated with this evolution equation. All these will be treated in this section. First, we design the feedback control. To simplify notations, let $y(x, t)=\tilde{y}\left(\ell x, \sqrt{\ell^{4} / E I \rho t}\right), I_{m}=\tilde{I}_{m} \ell^{-3} \rho^{-1}$, $M=\tilde{M} \ell^{-1} \rho^{-1}, J=\tilde{J} \ell^{-3} \rho^{-1}$. Then $y$ satisfies

$$
\left\{\begin{array}{l}
y_{t t}(x, t)+y_{x x x x}(x, t)=0, \quad 0<x<1, \quad t>0, \\
y(0, t)=0, \\
y_{x x}(0, t)-I_{m} y_{x t t}(0, t)+\ell^{2} E I^{-1} u\left(\sqrt{\ell^{4} / E I \rho t}\right)=0, \\
y_{x x x}(1, t)-M y_{t t}(1, t)=0, \\
y_{x x}(1, t)+J y_{x t t}(1, t)=0 .
\end{array}\right.
$$


We design a feedback controller:

$$
\ell^{2} E I^{-1} u\left(\sqrt{\ell^{4} / E I \rho t}\right)=-\alpha y_{x}(0, t)-\beta y_{x t}(0, t)+k y_{x x t}(0, t)
$$

where $\alpha>0$ and $\beta$ and $k$ are real numbers. Then the closed-loop system becomes

$$
\left\{\begin{array}{l}
y_{t t}(x, t)+y_{x x x x}(x, t)=0, \quad 0<x<1, t>0, \\
y(0, t)=0 \\
y_{x x}(0, t)-I_{m} y_{x t t}(0, t)-\alpha y_{x}(0, t)-\beta y_{x t}(0, t)+k y_{x x t}(0, t)=0, \\
y_{x x x}(1, t)-M y_{t t}(1, t)=0, \\
y_{x x}(1, t)+J y_{x t t}(1, t)=0 .
\end{array}\right.
$$

It seems not apparent that there is a Lyapunov function for the system above.

It should be pointed out that in deriving the third equation in (6), the motor driver of torque control type is used and the rate of change of the strain signal $y_{x x t}(0, t)$ is assumed being measurable. However, in practice, it is usually difficult to directly measure $y_{x x t}(0, t)$. In case $y_{x x t}(0, t)$ is not measurable, [9] suggests to use a control motor with a motor driver of speed reference type to get indirectly $y_{x x t}(0, t)$. Actually, from [9]

$$
V_{\text {ref }}(t) \doteq k_{f} y_{x t}(0, t)
$$

where $k_{f}>0$ is the back emf (electro-motive force) constant. "Back emf constant" specifies how much voltage is created by the armature conductors moving through the constant magnetic field in electrical motors. $V_{\text {ref }}(t)$ is the speed reference voltage of the control motor. Let $V_{\text {ref }}(t)$ be commanded to follow the following feedback law:

$$
V_{\mathrm{ref}}(t)=\frac{k_{f}}{I_{m}}\left[-\alpha \int_{0}^{t} y_{x}(0, \tau) d \tau-\beta y_{x}(0, t)+k y_{x x}(0, t)+\int_{0}^{t} y_{x x}(0, \tau) d \tau\right] .
$$

Note that the strain signal $y_{x x}(0, t)$ can be easily measured using strain gauges and hence the above feedback law is meaningful. 
Substituting such a $V_{\text {ref }}(t)$ into $V_{\text {ref }}(t)=k_{f} y_{x t}(0, t)$, we obtain also

$$
y_{x x}(0, t)-I_{m} y_{x t t}(0, t)-\alpha y_{x}(0, t)-\beta y_{x t}(0, t)+k y_{x x t}(0, t)=0 .
$$

Details can be found in Section V of [9].

Next, define the underlying state Hilbert space $H$ for the system (6): $H=H_{E}^{2}(0,1) \times L^{2}(0,1) \times \mathbb{C}^{3}, H_{E}^{2}(0,1)=\left\{f \in H^{2}(0,1), f(0)=0\right\}$ with the inner product induced norm

$$
\|(f, g, a, b, c)\|^{2}=\int_{0}^{1}\left[\left|f^{\prime \prime}(x)\right|^{2}+|g(x)|^{2}\right] d x+\alpha\left|f^{\prime}(0)\right|^{2}+\frac{|a|^{2}}{I_{m}}+\frac{|b|^{2}}{M}+\frac{|c|^{2}}{J}
$$

and the state variable

$$
Y(t)=\left(y(\cdot, t), y_{t}(\cdot, t), I_{m} y_{x t}(0, t)-k y_{x x}(0, t), M y_{t}(1, t), J y_{x t}(1, t)\right) .
$$

Then Eq. (6) is formulated to be an evolution equation in $H$ :

$$
\frac{d}{d t} Y(t)=A Y(t)
$$

where the associated system operator $A:(H \supset) D(A) \rightarrow H$ is defined as follows:

$$
\left\{\begin{array}{c}
A\left(\begin{array}{l}
\phi \\
\psi \\
a \\
b \\
c
\end{array}\right)=\left(\begin{array}{c}
\psi \\
-\phi^{(4)} \\
\phi^{\prime \prime}(0)-\alpha \phi^{\prime}(0)-\beta \psi^{\prime}(0) \\
\phi^{\prime \prime \prime}(1) \\
-\phi^{\prime \prime}(1),
\end{array}\right) \\
D(A)=\left\{(\phi, \psi, a, b, c) \in\left(H^{4} \cap H_{E}^{2}\right) \times H_{E}^{2} \times \mathbb{C}^{3} \mid\right. \\
\left.a=I_{m} \psi^{\prime}(0)-k \phi^{\prime \prime}(0), b=M \psi(1), c=J \psi^{\prime}(1)\right\}
\end{array}\right.
$$

Lemma $1 A^{-1}$ exists and is compact on $H$. Hence $\sigma(A)$, the spectrum of $A$, consists of isolated eigenvalues only.

Proof For any $(f, g, a, b, c) \in H$, solving

$$
A\left(\begin{array}{c}
\phi \\
\psi \\
I_{M} \psi^{\prime}(0)-k \phi^{\prime \prime}(0) \\
M \psi(1) \\
J \psi^{\prime}(1)
\end{array}\right)=\left(\begin{array}{c}
\psi \\
-\phi^{(4)} \\
\phi^{\prime \prime}(0)-\alpha \phi^{\prime}(0)-\beta \psi^{\prime}(0) \\
\phi^{\prime \prime \prime}(1) \\
-\phi^{\prime \prime}(1)
\end{array}\right)=\left(\begin{array}{l}
f \\
g \\
a \\
b \\
c
\end{array}\right)
$$


produces the unique solution (notice that $\phi(0)=0) \psi=f \in H_{E}^{2}$ and $\phi \in H^{4} \cap H_{E}^{2}$

$$
\begin{aligned}
\phi(x)= & -\left[\frac{a+\beta f^{\prime}(0)}{\alpha}+\frac{c}{\alpha}+\frac{2 b+b \alpha}{2 \alpha}+\frac{1}{2} \int_{0}^{1} s^{2} g(s) d s+\frac{1}{\alpha} \int_{0}^{1} s g(s) d s\right] x \\
& +\frac{b}{6}+\int_{0}^{1} \frac{1}{6} s^{3} g(s) d s-\frac{c}{2} x^{2}+\frac{b}{6}(x-1)^{3}-\frac{1}{6} \int_{1}^{x}(x-s)^{3} g(s) d s .
\end{aligned}
$$

The result then follows from the Sobolev's embedding theorem ([17], p. 208).

Lemma 2 For any $\lambda=i \tau^{2} \in \sigma(A)$, there is a unique eigenfunction (up to a scalar)

$$
\begin{aligned}
& \left(\phi, \lambda \phi, I_{m} \lambda \phi^{\prime}(0)-k \phi^{\prime \prime}(0), M \lambda \phi(1), J \lambda \phi^{\prime}(1)\right) \\
& \quad=\left(\phi, \lambda \phi, \lambda^{-1}\left[\phi^{\prime \prime}(0)-(\beta \lambda+\alpha) \phi^{\prime}(0)\right], \lambda^{-1} \phi^{\prime \prime \prime}(1),-\lambda^{-1} \phi^{\prime \prime}(1)\right)
\end{aligned}
$$

where

$$
\begin{aligned}
\phi(x)= & -\left(1+M J \tau^{4}\right) \sinh \tau x \\
& +\left[-2 J \tau^{3} \cos \tau+\left(-1+M J \tau^{4}\right) \sin \tau\right] \cosh \tau(1-x) \\
& +\left[2 J \tau^{3} \cosh \tau-\left(1+M J \tau^{4}\right) \sin \tau+\left(-1+M J \tau^{4}\right) \sinh \tau\right] \cos \tau(1-x) \\
& +\left[\left(1-M J \tau^{4}\right) \cos \tau-2 M \tau \sin \tau\right] \sinh \tau(1-x) \\
& +\left[\left(1-M J \tau^{4}\right) \cosh \tau+\left(1+M J \tau^{4}\right) \cos \tau+2 M \tau \sinh \tau\right] \sin \tau(1-x)
\end{aligned}
$$

and the characteristic equation that $\lambda$ satisfies is

$$
\begin{aligned}
& \left(-I_{m} \tau^{4}+i \beta \tau^{2}+\alpha\right)\left\{1+M J \tau^{4}+\left(M \tau-J \tau^{3}\right) \sinh \tau \cos \tau\right. \\
& \left.-\left(M \tau+J \tau^{3}\right) \cosh \tau \sin \tau+\left(1-M J \tau^{4}\right) \cosh \tau \cos \tau\right\} \\
& +\tau\left(k i \tau^{2}+1\right)\left\{-2 J \tau^{3} \cosh \tau \cos \tau+\left(-1+M J \tau^{4}\right) \cosh \tau \sin \tau\right. \\
& \left.+\left(1-M J \tau^{4}\right) \sinh \tau \cos \tau-2 M \tau \sinh \tau \sin \tau\right\}=0 .
\end{aligned}
$$


Proof Solving the eigenvalue problem

$$
A\left(\begin{array}{l}
\phi \\
\psi \\
a \\
b \\
c
\end{array}\right)=\left(\begin{array}{c}
\psi \\
-\phi^{(4)} \\
\phi^{\prime \prime}(0)-\alpha \phi^{\prime}(0)-\beta \psi^{\prime}(0) \\
\phi^{\prime \prime \prime}(1) \\
-\phi^{\prime \prime}(1)
\end{array}\right)=\lambda\left(\begin{array}{c}
\phi \\
\psi \\
I_{m} \psi^{\prime}(0)-k \phi^{\prime \prime}(0) \\
M \psi(1) \\
J \psi^{\prime}(1)
\end{array}\right)
$$

one has $\psi=\lambda \phi$ and

$$
\left\{\begin{array}{l}
\phi^{\prime \prime \prime \prime}+\lambda^{2} \phi=0 \\
\phi(0)=\left(I_{m} \lambda^{2}+\beta \lambda+\alpha\right) \phi^{\prime}(0)-(k \lambda+1) \phi^{\prime \prime}(0)=0 \\
\phi^{\prime \prime \prime}(1)-M \lambda^{2} \phi(1)=0 \\
\phi^{\prime \prime}(1)+J \lambda^{2} \phi^{\prime}(1)=0 .
\end{array}\right.
$$

Hence

$$
\begin{aligned}
& \left(I_{m} \lambda \phi^{\prime}(0)-k \phi^{\prime \prime}(0), M \lambda \phi(1), J \lambda \phi^{\prime}(1)\right) \\
& \quad=\left(\lambda^{-1}\left[\phi^{\prime \prime}(0)-(\beta \lambda+\alpha) \phi^{\prime}(0)\right], \lambda^{-1} \phi^{\prime \prime \prime}(1),-\lambda^{-1} \phi^{\prime \prime}(1)\right) .
\end{aligned}
$$

Let $f(x)=\phi(1-x)$. Then $f$ satisfies

$$
\left\{\begin{array}{l}
f^{\prime \prime \prime \prime}+\lambda^{2} f=0 \\
f(1)=\left(I_{m} \lambda^{2}+\beta \lambda+\alpha\right) f^{\prime}(1)+(k \lambda+1) f^{\prime \prime}(1)=0 \\
f^{\prime \prime \prime}(0)+M \lambda^{2} f(0)=0 \\
f^{\prime \prime}(0)-J \lambda^{2} f^{\prime}(0)=0 .
\end{array}\right.
$$

Let $\lambda=i \tau^{2}$. It is easily seen that for any $\lambda=i \tau^{2}$, the general solution of the following equation

$$
\left\{\begin{array}{l}
f^{\prime \prime \prime \prime}+\lambda^{2} f=0, \\
f^{\prime \prime \prime}(0)+M \lambda^{2} f(0)=0, \\
f^{\prime \prime}(0)-J \lambda^{2} f^{\prime}(0)=0 .
\end{array}\right.
$$


is of the form

$$
\begin{aligned}
f(x)= & {\left[\left(c_{1}-c_{2}\right)-M J \tau^{4}\left(c_{1}+c_{2}\right)\right] \cosh \tau x } \\
& +\left[\left(c_{1}-c_{2}\right)+M J \tau^{4}\left(c_{1}+c_{2}\right)\right] \cos \tau x \\
& +2 M \tau\left(c_{1} \sinh \tau x+c_{2} \sin \tau x\right)
\end{aligned}
$$

where $c_{1}, c_{2}$ are arbitrary constants. By $f(1)=0$, one has (up to a scalar)

$$
\begin{aligned}
c_{1} & =\left(1+M J \tau^{4}\right) \cosh \tau+\left(1-M J \tau^{4}\right) \cos \tau-2 M \tau \sin \tau, \\
c_{2} & =\left(1-M J \tau^{4}\right) \cosh \tau+\left(1+M J \tau^{4}\right) \cos \tau+2 M \tau \sinh \tau, \\
c_{1}-c_{2} & =2 M J \tau^{4} \cosh \tau-2 M J \tau^{4} \cos \tau-2 M \tau \sin \tau-2 M \tau \sinh \tau, \\
c_{1}+c_{2} & =2 \cosh \tau+2 \cos \tau-2 M \tau \sin \tau+2 M \tau \sinh \tau .
\end{aligned}
$$

Hence (again up to a scalar)

$$
\begin{aligned}
f(x)= & -\left(1+M J \tau^{4}\right) \sinh \tau(1-x) \\
& +\left[-2 J \tau^{3} \cos \tau+\left(-1+M J \tau^{4}\right) \sin \tau\right] \cosh \tau x \\
& +\left[2 J \tau^{3} \cosh \tau-\left(1+M J \tau^{4}\right) \sin \tau+\left(-1+M J \tau^{4}\right) \sinh \tau\right] \cos \tau x \\
& +\left[\left(1-M J \tau^{4}\right) \cos \tau-2 M \tau \sin \tau\right] \sinh \tau x \\
& +\left[\left(1-M J \tau^{4}\right) \cosh \tau+\left(1+M J \tau^{4}\right) \cos \tau+2 M \tau \sinh \tau\right] \sin \tau x
\end{aligned}
$$

this is (12) by $\phi(x)=f(1-x)$. Note that the function $f$ defined above actually satisfies

$$
\left\{\begin{array}{l}
f^{\prime \prime \prime \prime}+\lambda^{2} f=0 \\
f(1)=0 \\
f^{\prime \prime \prime}(0)+M \lambda^{2} f(0)=0 \\
f^{\prime \prime}(0)-J \lambda^{2} f^{\prime}(0)=0
\end{array}\right.
$$

for any $\lambda=i \tau^{2}$. In order $f$ (it is obvious that $f$ cannot be identical zero) to be a solution of (15), it is necessary and sufficient that $\left(I_{m} \lambda^{2}+\beta \lambda+\alpha\right) f^{\prime}(1)+(k \lambda+1) f^{\prime \prime}(1)=0$ which induces (13), proving the lemma. 
Lemma 3 There is a family of eigenvalues $\left\{\lambda_{n}=i \tau_{n}^{2},-i \bar{\tau}_{n}^{2}\right\}$ of $A$ with the following asymptotic expression

$$
\lambda_{n}=i \tau_{n}^{2}=-k I_{m}^{-1}+i\left[M^{-1}+(m \pi)^{2}\right]+\mathcal{O}\left(n^{-1}\right)
$$

where $m=n-1 / 2, n$ is a sufficiently large positive integer. A corresponding eigenfunction

$$
\begin{aligned}
\Phi_{n} & =\left(\phi_{n}, \lambda_{n} \phi_{n}, I_{m} \lambda_{n} \phi_{n}^{\prime}(0)-k \phi_{n}^{\prime \prime}(0), M \lambda_{n} \phi_{n}(1), J \lambda_{n} \phi_{n}^{\prime}(1)\right) \\
& =\left(\phi_{n}, \lambda_{n} \phi_{n}, \lambda_{n}^{-1}\left[\phi_{n}^{\prime \prime}(0)-\left(\beta \lambda_{n}+\alpha\right) \phi_{n}^{\prime}(0)\right], \lambda_{n}^{-1} \phi_{n}^{\prime \prime \prime}(1),-\lambda_{n}^{-1} \phi_{n}^{\prime \prime}(1)\right)
\end{aligned}
$$

where

$$
\begin{aligned}
\phi_{n}(x)= & -\left(1+M J \tau^{4}\right) \sinh \tau_{n} x \\
+ & {\left[-2 J \tau_{n}^{3} \cos \tau_{n}+\left(-1+M J \tau_{n}^{4}\right) \sin \tau_{n}\right] \cosh \tau_{n}(1-x) } \\
+ & {\left[2 J \tau_{n}^{3} \cosh \tau_{n}-\left(1+M J \tau_{n}^{4}\right) \sin \tau_{n}\right.} \\
& \left.+\left(-1+M J \tau_{n}^{4}\right) \sinh \tau_{n}\right] \cos \tau_{n}(1-x) \\
+ & {\left[\left(1-M J \tau_{n}^{4}\right) \cos \tau_{n}-2 M \tau_{n} \sin \tau_{n}\right] \sinh \tau_{n}(1-x) } \\
+ & {\left[\left(1-M J \tau_{n}^{4}\right) \cosh \tau_{n}+\left(1+M J \tau_{n}^{4}\right) \cos \tau_{n}\right.} \\
& \left.+2 M \tau_{n} \sinh \tau_{n}\right] \sin \tau_{n}(1-x)
\end{aligned}
$$

which is obtained by (12) with $\tau=\tau_{n}$. The following asymptotic expression holds

$$
\begin{aligned}
F_{n}(x)= & \left(\begin{array}{c}
e^{-m \pi(1-x)}-e^{-m \pi x} \sin m \pi+\cos m \pi(1-x)-\sin m \pi(1-x) \\
i\left[e^{-m \pi(1-x)}-e^{-m \pi x} \sin m \pi-\cos m \pi(1-x)+\sin m \pi(1-x)\right] \\
0 \\
0 \\
0
\end{array}\right) \\
& +\mathcal{O}\left(n^{-1}\right)
\end{aligned}
$$

where

$$
\begin{aligned}
F_{n}(x)= & -2(M J)^{-1} \tau_{n}^{-6} e^{-\tau_{n}} \\
& \times\left(\phi_{n}^{\prime \prime}, \lambda_{n} \phi_{n}, I_{m} \lambda_{n} \phi_{n}^{\prime}(0)-k \phi_{n}^{\prime \prime}(0), M \lambda_{n} \phi_{n}(1), J \lambda_{n} \phi_{n}^{\prime}(1)\right)^{T} .
\end{aligned}
$$


(18) holds uniformly in $x \in[0,1]$. It is seen that

$$
\lim _{n \rightarrow \infty}\left\|F_{n}\right\|_{L^{2} \times L^{2} \times \mathbb{C}^{3}}^{2}=\lim _{n \rightarrow \infty}\left\|2(M J)^{-1} \tau_{n}^{-6} e^{-\tau_{n}} \Phi_{n}\right\|_{H}^{2}=2 .
$$

Proof Note that for a large positive integer $n$, in a uniformly bounded small neighborhood of $m \pi=(n-1 / 2) \pi$,

$$
|\sin \tau| \leq C, \quad|\cos \tau| \leq C, \quad\left|e^{-\tau} \sinh \tau\right| \leq C, \quad\left|e^{-\tau} \cosh \tau\right| \leq C
$$

uniformly for all $n$ with some constant $C$. By multiplying $\left(-I_{m} M J\right)^{-1} \tau^{-8} e^{-\tau}$ on both sides of (13), we can write (13), in a uniformly bounded small neighborhood of $m \pi=(n-1 / 2) \pi$ for each $n$, to be

$$
\cos \tau=\mathcal{O}\left(|\tau|^{-1}\right)
$$

or

$$
\cos \tau=-\frac{1}{\tau}\left(M^{-1}+i k I_{m}^{-1}\right) \sin \tau+\mathcal{O}\left(|\tau|^{-2}\right)
$$

Applying the Rouche's theorem ([18], p. 181) to two functions $\cos \tau$ and $-\mathcal{O}\left(|\tau|^{-1}\right)$ in a small neighborhood of $m \pi$ for each $n$, we can obtain a solution $\tau_{n}$ of (19):

$$
\tau=\tau_{n}=(n-1 / 2) \pi+\mathcal{O}\left(n^{-1}\right)
$$

for sufficiently large $n$. Substituting (21) into (20) yields

$$
\mathcal{O}\left(n^{-1}\right)=\frac{1}{(n-1 / 2) \pi}\left(M^{-1}+i k I_{m}^{-1}\right)+\mathcal{O}\left(n^{-2}\right)
$$

and so

$$
\tau_{n}=(n-1 / 2) \pi+\frac{1}{(n-1 / 2) \pi}\left(M^{-1}+i k I_{m}^{-1}\right)+\mathcal{O}\left(n^{-2}\right)
$$

hence

$$
\lambda_{n}=i \tau_{n}^{2}=-k I_{m}^{-1}+i\left[M^{-1}+((n-1 / 2) \pi)^{2}\right]+\mathcal{O}\left(n^{-1}\right) .
$$


For the estimation of (18), we treat the first component only because the second component can be treated similarly and $\lambda_{n}^{-1} \phi_{n}^{\prime \prime}(0), \lambda_{n}^{-1} \phi_{n}^{\prime \prime}(1)$, $\lambda_{n}^{-1} \phi_{n}^{\prime \prime \prime}(1)$ can be found first from the expression below and then treated in a similar fashion. Now

$$
\begin{aligned}
\tau_{n}^{-2} \phi_{n}^{\prime \prime}(x)= & -\left(1+M J \tau^{4}\right) \sinh \tau_{n} x \\
+ & {\left[-2 J \tau_{n}^{3} \cos \tau_{n}+\left(-1+M J \tau_{n}^{4}\right) \sin \tau_{n}\right] \cosh \tau_{n}(1-x) } \\
- & {\left[2 J \tau_{n}^{3} \cosh \tau_{n}-\left(1+M J \tau_{n}^{4}\right) \sin \tau_{n}\right.} \\
& \left.+\left(-1+M J \tau_{n}^{4}\right) \sinh \tau_{n}\right] \cos \tau_{n}(1-x) \\
+ & {\left[\left(1-M J \tau_{n}^{4}\right) \cos \tau_{n}-2 M \tau_{n} \sin \tau_{n}\right] \sinh \tau_{n}(1-x) } \\
- & {\left[\left(1-M J \tau_{n}^{4}\right) \cosh \tau_{n}+\left(1+M J \tau_{n}^{4}\right) \cos \tau_{n}\right.} \\
& \left.+2 M \tau_{n} \sinh \tau_{n}\right] \sin \tau_{n}(1-x) .
\end{aligned}
$$

Since for any bounded $y>0$ and $x \in[0,1]$, it holds uniformly

$$
\begin{gathered}
e^{-\tau_{n} y}=e^{-m \pi y}+\mathcal{O}\left(n^{-1}\right), \\
\sin \tau_{n} x=\sin m \pi x+\mathcal{O}\left(n^{-1}\right), \quad \cos \tau_{n} x=\cos m \pi x+\mathcal{O}\left(n^{-1}\right) .
\end{gathered}
$$

Hence

$$
\begin{aligned}
2(M J)^{-1} \tau_{n}^{-6} e^{-\tau_{n}} \phi_{n}^{\prime \prime}(x)= & -e^{-\tau_{n}(1-x)}+e^{-\tau_{n} x} \sin \tau_{n}-\cos \tau_{n}(1-x) \\
& +\sin \tau_{n}(1-x)+\mathcal{O}\left(n^{-1}\right) \\
= & -e^{-m \pi(1-x)}+e^{-m \pi x} \sin m \pi-\cos m \pi(1-x) \\
& +\sin m \pi(1-x)+\mathcal{O}\left(n^{-1}\right)
\end{aligned}
$$

Moreover,

$$
2(M J)^{-1} \tau_{n}^{-6} e^{-\tau_{n}} \phi_{n}^{\prime}(0)=\mathcal{O}\left(n^{-1}\right) .
$$

This is the result required.

It should be pointed out that up to now, we can only say that (17) and (18) are valid for "a family of eigenpairs" of $A$ only. However, in the next section, we shall show that they are indeed asymptotic expressions of all eigenpairs of $A$. This is one of merits of the approach. 


\section{RIESZ BASIS PROPERTY}

Let us recall that for a closed linear operator $\mathbf{A}$ in a Hilbert space $\mathbf{H}$, a nonzero $x \in \mathbf{H}$ is called a generalized eigenvector of $\mathbf{A}$, corresponding to an eigenvalue $\lambda$ of $\mathbf{A}$ that has finite algebraic multiplicity, if there is a positive integer $n$ such that $(\lambda-\mathbf{A})^{n} x=0$. A sequence $\left\{x_{n}\right\}_{n=1}^{\infty}$ in $\mathbf{H}$ is called a Riesz basis for $\mathbf{H}$ if there is an orthonormal basis $\left\{e_{n}\right\}_{n=1}^{\infty}$ in $\mathbf{H}$ and a linear bounded invertible operator $\mathbf{T}$ such that

$$
\mathbf{T} e_{n}=x_{n}, \quad n=1,2, \ldots
$$

It is seen that each Riesz basis sequence must be approximately normalized:

$$
C_{1} \leq\left\|x_{n}\right\| \leq C_{2}, \quad C_{1}, C_{2}>0, n=1,2, \ldots
$$

Suppose that $\left\{\lambda_{n}\right\}_{n=1}^{\infty} \subset \sigma(\mathbf{A})$. If each $\lambda_{n}$ has finite algebraic multiplicity $m_{n}$, then there is a sequence of linear independent generalized eigenvectors $\left\{x_{n_{i}}\right\}_{i=1}^{m_{n}}$ corresponding to $\lambda_{n}$. If $m_{n}=1$ for sufficiently large $n$ and $\left\{\left\{x_{n_{i}}\right\}_{i=1}^{m_{n}}\right\}_{n=1}^{\infty}$ forms a Riesz basis for $\mathbf{H}$, then $\mathbf{A}$ generates a $C_{0^{-}}$ semigroup $e^{\mathbf{A} t}$ which can be represented as

$$
e^{\mathbf{A} t} x=\sum_{n=1}^{\infty} e^{\lambda_{n} t} \sum_{i=1}^{m_{n}} a_{n i} \sum_{j=1}^{m_{n}} f_{n i j}(t) x_{n_{j}}, \quad \text { for any } x=\sum_{n=1}^{\infty} \sum_{i=1}^{m_{n}} a_{n i} x_{n_{i}} \in \mathbf{H}
$$

where $f_{n i j}(t)$ is a polynomial of $t$ with order less than $m_{n}$. In particular, if $a<\operatorname{Re} \lambda<b$ for some reals $a$ and $b$ then $\mathbf{A}$ generates a $C_{0}$-group on H. Moreover, the spectrum-determined growth condition holds for $e^{\mathbf{A} t}: \omega(\mathbf{A})=S(\mathbf{A})$, where $\omega(\mathbf{A})$ is the growth order of $e^{\mathbf{A} t}$ and $S(\mathbf{A})$ is the spectral bound of $e^{\mathbf{A} t}$.

The following result is recently reported in [8]:

THEOREM 1 Let $\mathbf{A}$ be a densely defined discrete operator (that is, $(\lambda-\mathbf{A})^{-1}$ is compact for some $\left.\lambda\right)$ in a Hilbert space $\mathbf{H}$. Let $\left\{z_{n}\right\}_{n=1}^{\infty}$ be a Riesz basis for $\mathbf{H}$. If there exist an $N \geq 0$ and a sequence of generalized 
eigenvectors $\left\{x_{n}\right\}_{n=N+1}^{\infty}$ of $\mathbf{A}$ such that

$$
\sum_{N+1}^{\infty}\left\|x_{n}-z_{n}\right\|^{2}<\infty
$$

then

(i) there exists a constant $M>N$ and generalized eigenvectors $\left\{x_{n 0}\right\}_{n=1}^{M}$ of $\mathbf{A}$ such that $\left\{x_{n 0}\right\}_{n=1}^{M} \cup\left\{x_{n}\right\}_{n=M+1}^{\infty}$ forms a Riesz basis for $\mathbf{H}$;

(ii) let $\left\{x_{n 0}\right\}_{n=1}^{M} \cup\left\{x_{n}\right\}_{n=M+1}^{\infty}$ correspond to eigenvalues $\left\{\sigma_{n}\right\}_{n=1}^{\infty}$ of $\mathbf{A}$. Then $\sigma(\mathbf{A})=\left\{\sigma_{n}\right\}_{n=1}^{\infty}$, where $\sigma_{n}$ is counted according to its algebraic multiplicity;

(iii) if there is an $M_{0}>0$ such that $\sigma_{n} \neq \sigma_{m}$ for all $m, n>M_{0}$, then there exists an $N_{0}>M_{0}$ such that all $\sigma_{n}, n>N_{0}$ are algebraically simple.

In Lemma 3, we have found a sequence of approximate normalized generalized eigenfunctions of $A$. In order to apply Theorem 1, we have to find a reference Riesz basis. This is realized by finding a discrete skew-adjoint linear operator in $H$. From functional analysis, for a discrete skew-adjoint linear operator in $H$, there is always a sequence of generalized eigenvectors which forms a Riesz basis, moreover, for such an operator, the geometric multiplicity of each eigenvalue is identical to its algebraic multiplicity and all eigenvalues lie on the imaginary axis.

Now we show the game. Let operator $A_{0}$ be the operator $A$ with $\beta=k=0$. That is,

$$
\left\{\begin{array}{c}
A_{0}\left(\begin{array}{l}
\phi \\
\psi \\
a \\
b \\
c
\end{array}\right)=\left(\begin{array}{c}
\psi \\
-\phi^{(4)} \\
\phi^{\prime \prime}(0)-\alpha \phi^{\prime}(0) \\
\phi^{\prime \prime \prime}(1) \\
-\phi^{\prime \prime}(1)
\end{array}\right) \\
D\left(A_{0}\right)=\left\{(\phi, \psi, a, b, c) \in\left(H^{4} \cap H_{E}^{2}\right) \times H_{E}^{2} \times \mathbb{C}^{3} \mid a=I_{m} \psi^{\prime}(0),\right. \\
\left.b=M \psi(1), c=J \psi^{\prime}(1)\right\}
\end{array}\right.
$$

Then it is easily checked that $A_{0}$ is indeed a discrete skew-adjoint linear operator in $H$. Because in previous sections, we consider $\beta$ 
and $k$ being only real numbers, this means that all results in previous sections are still valid for $A_{0}$. From Lemma 2, each eigenvalue of $A_{0}$ is geometrically simple and hence algebraically simple and the characteristic equation satisfied by $\mu=i \omega^{2} \in \sigma\left(A_{0}\right)$ is

$$
\begin{aligned}
& \left(-I_{m} \omega^{4}+\alpha\right)\left\{1+M J \omega^{4}+\left(M \omega-J \omega^{3}\right) \sinh \omega \cos \omega\right. \\
& \left.-\left(M \omega+J \omega^{3}\right) \cosh \omega \sin \omega+\left(1-M J \omega^{4}\right) \cosh \omega \cos \omega\right\} \\
& +\omega\left\{-2 J \omega^{3} \cosh \omega \cos \omega+\left(-1+M J \omega^{4}\right) \cosh \omega \sin \omega\right. \\
& \left.+\left(1-M J \omega^{4}\right) \sinh \omega \cos \omega-2 M \omega \sinh \omega \sin \omega\right\}=0 .
\end{aligned}
$$

Because all eigenvalues of $A_{0}$ lie on the imaginary axis and the eigenvalues appear in conjugate pairs, we need consider only positive solutions of (23) in order to find eigenvalues of $A_{0}$. Like (19), we write (23) asymptotically to be

$$
\cos \omega=\mathcal{O}\left(\omega^{-1}\right)
$$

The positive solutions of (24) are of

$$
\omega=\omega_{n}=(n-1 / 2) \pi+\mathcal{O}\left(n^{-1}\right)
$$

for sufficiently large $n$. Same to (17), it has

$$
\mu_{n}=i \omega_{n}^{2}=i\left[M^{-1}+(m \pi)^{2}\right]+\mathcal{O}\left(n^{-1}\right) .
$$

The difference between (26) and (17) is that (26) is indeed an asymptotic expression for all eigenvalues of $A_{0}$ on the up half complex plane.

From Lemma 2, we can obtain the unique (up to a scalar) eigenfunction of $A_{0}$ associated with $\mu_{n}$ to be

$$
\begin{aligned}
\Psi_{n} & =\left(f_{n}, \mu_{n} f_{n}, I_{m} \mu_{n} f_{n}^{\prime}(0), M \mu_{n} f_{n}(1), J \mu_{n} f_{n}^{\prime}(1)\right) \\
& =\left(f_{n}, \mu_{n} f_{n}, \mu_{n}^{-1}\left[f_{n}^{\prime \prime}(0)-\alpha f_{n}^{\prime}(0)\right], \mu_{n}^{-1} f_{n}^{\prime \prime \prime}(1),-\mu_{n}^{-1} f_{n}^{\prime \prime}(1)\right)
\end{aligned}
$$


where

$$
\begin{aligned}
f_{n}(x)=- & \left(1+M J \omega^{4}\right) \sinh \omega_{n} x \\
+ & {\left[-2 J \omega_{n}^{3} \cos \omega_{n}+\left(-1+M J \omega_{n}^{4}\right) \sin \omega_{n}\right] \cosh \omega_{n}(1-x) } \\
+ & {\left[2 J \omega_{n}^{3} \cosh \omega_{n}-\left(1+M J \omega_{n}^{4}\right) \sin \omega_{n}\right.} \\
& \left.+\left(-1+M J \omega_{n}^{4}\right) \sinh \omega_{n}\right] \cos \omega_{n}(1-x) \\
+ & {\left[\left(1-M J \omega_{n}^{4}\right) \cos \omega_{n}-2 M \omega_{n} \sin \omega_{n}\right] \sinh \omega_{n}(1-x) } \\
+ & {\left[\left(1-M J \omega_{n}^{4}\right) \cosh \omega_{n}+\left(1+M J \omega_{n}^{4}\right) \cos \omega_{n}\right.} \\
& \left.+2 M \omega_{n} \sinh \omega_{n}\right] \sin \omega_{n}(1-x) .
\end{aligned}
$$

By Lemma 3

$$
\begin{aligned}
G_{n}(x)= & \left(\begin{array}{c}
e^{-m \pi(1-x)}-e^{-m \pi x} \sin m \pi+\cos m \pi(1-x)-\sin m \pi(1-x) \\
i\left[e^{-m \pi(1-x)}-e^{-m \pi x} \sin m \pi-\cos m \pi(1-x)+\sin m \pi(1-x)\right] \\
0 \\
0 \\
0
\end{array}\right) \\
& +\mathcal{O}\left(n^{-1}\right)
\end{aligned}
$$

where

$G_{n}(x)=-2(M J)^{-1} \omega_{n}^{-6} e^{-\omega_{n}}\left(f_{n}^{\prime \prime}, \mu_{n} f_{n}, I_{m} \mu_{n} f_{n}^{\prime}(0), M \mu_{n} f_{n}(1), J \mu_{n} f_{n}^{\prime}(1)\right)^{T}$

and

$$
-2(M J)^{-1} \omega_{n}^{-6} e^{-\omega_{n}} f_{n}^{\prime}(0)=\mathcal{O}\left(n^{-1}\right)
$$

Since $A_{0}$ is a discrete operator, there are only finite number of eigenvalues in any bounded complex region, all $\left\{-2(M J)^{-1} \times\right.$ $\left.\omega_{n}^{-6} e^{-\omega_{n}} \Psi_{n}\right\} \cup\{$ their conjugates $\}$ with at most other finite number of generalized eigenfunctions (in the sense of $\omega$-linearly independent) of $A_{0}$ form a Riesz basis for $H$. Therefore, we may assume, without loss of generality that

Generalized eigenfunctions of $A_{0}=\left\{-2(M J)^{-1} \omega_{n}^{-6} e^{-\omega_{n}} \Psi_{n}\right\}_{1}^{\infty} \cup$ \{their conjugates\}. 
It follows from (18) and (29) that there exists an $N>0$ such that

$$
\sum_{n>N}^{\infty}\left\|2(M J)^{-1} \tau_{n}^{-6} e^{-\tau_{n}} \Phi_{n}-2(M J)^{-1} \omega_{n}^{-6} e^{-\omega_{n}} \Psi_{n}\right\|_{H}^{2}=\sum_{n>N}^{\infty} \mathcal{O}\left(n^{-2}\right)<\infty
$$

The same thing is true for their conjugates. Therefore, considering $\left\{-\Psi_{n} /\left(M J \omega_{n}^{6} e^{\omega_{n}}\right)\right\}_{1}^{\infty} \cup\{$ their conjugates $\}$ being a reference Riesz basis, Theorem 1 can be applied to obtain our main result of the article.

THEOREM 2 For any $\alpha>0$ and real numbers $\beta$ and $k$

(i) there is a sequence of generalized eigenfunctions of operator $A$, which forms a Riesz basis for the state space $H$;

(ii) (17) is an asymptotic expression for all eigenvalues of $A$;

(iii) all eigenvalues of $A$ with sufficiently large modulus are algebraically simple, therefore, $A$ generates a $C_{0}$-group on $H$ and the spectrumdetermined growth condition holds for the semigroup $e^{A t}$ generated by $A: \omega(A)=S(A)$.

\section{EXPONENTIAL STABILITY}

It is seen from (17) that if $k<0$, then system (6) is never exponentially stable. In this section, we shall show that system (6) is exponentially stable if

$$
\beta \geq \alpha k>0
$$

Since the spectrum-determined growth condition holds, it follows from (ii) of Theorem 2 that $e^{A t}$ is exponentially stable under condition (31) if and only if $\operatorname{Re} \lambda<0$ for all $\lambda \in \sigma(A)$.

TheOREm 3 Suppose $\beta \geq \alpha k>0$. Then there exists an $\omega>0$ such that $\operatorname{Re} \lambda<-\omega$ for all $\lambda \in \sigma(A)$. Therefore, the $C_{0}$-semigroup $e^{\text {At }}$ generated by $A$ is exponentially stable:

$$
\left\|e^{A t} \Phi\right\| \leq M e^{-\omega t}\|\Phi\|, \quad \text { for any } \Phi \in H
$$

where $M>0$ is a constant independent of $\Phi$. 
Proof It suffices to show that $\operatorname{Re} \lambda<0$ for all $\lambda \in \sigma(A)$. We start from the eigen problem (14). Assume $k \lambda+1 \neq 0$. Multiplying $\bar{\phi}$, the conjugate of $\phi$, on both sides of the first equation in (14) and integrating from 0 to 1 with respect to $x$ yields

$$
\begin{aligned}
& \int_{0}^{1}\left|\phi^{\prime \prime}(x)\right|^{2} d x+\lambda^{2}\left[\int_{0}^{1}|\phi(x)|^{2} d x+M|\phi(1)|^{2}+J\left|\phi^{\prime}(1)\right|^{2}\right] \\
& +\frac{I_{m} \lambda^{2}+\beta \lambda+\alpha}{k \lambda+1}\left|\phi^{\prime}(0)\right|^{2}=0 .
\end{aligned}
$$

Clearly, if $\lambda$ is a real number, it must have $\lambda<0$ (Notice Lemma 1, $\lambda=0$ is always not in the spectrum of $A$ ). Suppose that $\lambda=\lambda_{1}+i \lambda_{2}$, $\lambda_{2} \neq 0$. Then comparing the imaginary part of (32) yields

$$
\begin{array}{r}
2 \lambda_{1}\left[\int_{0}^{1}|\phi(x)|^{2} d x+M|\phi(1)|^{2}+J\left|\phi^{\prime}(1)\right|^{2}\right] \\
+\frac{k I_{m}|\lambda|^{2}+2 I_{m} \lambda_{1}+\beta-\alpha k}{|k \lambda+1|^{2}}\left|\phi^{\prime}(0)\right|^{2}=0 .
\end{array}
$$

There are two cases. When $\lambda_{1} \neq 0$, it is obvious that $\lambda_{1}<0$ as $\beta \geq \alpha k$. While as $\lambda_{1}=0$, it must be $\phi^{\prime}(0)=0$ and so $\phi^{\prime \prime}(0)=0$ from the boundary condition of (14). In this case, the solution of (14) shall be (we may assume that $\left.\lambda_{2}>0\right) \quad \phi(x)=\sinh \sqrt{\lambda_{2} x}-\sin \sqrt{\lambda_{2} x}$. But from the boundary condition $\phi^{\prime \prime \prime}(1)=-M \lambda_{2}^{2} \phi(1)$, we arrive the contradiction that

$$
\cosh \sqrt{\lambda_{2}}+\cos \sqrt{\lambda_{2}}=-M \sqrt{\lambda_{2}}\left(\sinh \sqrt{\lambda_{2}}-\sin \sqrt{\lambda_{2}}\right) .
$$

The proof is complete.

\section{EXACT CONTROLLABILITY}

In this section, we consider the following control problem:

$$
\left\{\begin{array}{l}
y_{t t}(x, t)+y_{x x x x}(x, t)=0, \quad 0<x<1, \quad t>0, \\
y(0, t)=0 \\
y_{x x}(0, t)-I_{m} y_{x t t}(0, t)-\alpha y_{x}(0, t)+v(t)=0, \\
y_{x x x}(1, t)-M y_{t t}(1, t)=0 \\
y_{x x}(1, t)+J y_{x t t}(1, t)=0 .
\end{array}\right.
$$


Let

$$
Z(t)=\left(y(\cdot, t), y_{t}(\cdot, t), I_{m} y_{x t}(0, t), M y_{t}(1, t), J y_{x t}(1, t)\right) .
$$

In the space $H$, we can write (34) as

$$
\frac{d}{d t} Z(t)=A_{0} Z(t)+b v(t), \quad b=(0,0,1,0,0) \in H
$$

where $A_{0}$ is defined by (22). For simplicity, we denote the all eigenfunctions of $A_{0}$ as following:

$$
\left\{\tilde{\Psi}_{n}\right\}_{n \in \mathbb{Z}}=\left\{\left(g_{n}, \mu_{n} g_{n}, I_{m} \mu_{n} g_{n}^{\prime}(0), M \mu_{n} g_{n}(1), J \mu_{n} g_{n}^{\prime}(1)\right)\right\}_{n \in \mathbb{Z}}
$$

which satisfies

$$
\left(g_{n}^{\prime \prime}, \mu_{n} g_{n}, I_{m} \mu_{n} g_{n}^{\prime}(0), M \mu_{n} g_{n}(1), J \mu_{n} g_{n}^{\prime}(1)\right)=G_{n}(x)^{T}, \quad g_{n}^{\prime}(0)=\mathcal{O}\left(n^{-1}\right)
$$

where $G_{n}$ is defined by (29).

Definition 1 System (36) (or $\left(A_{0}, b\right)$ ) is called exact controllable on $[0, T]$ in $H$ if for any given $Z_{0}, Z_{1} \in H$, there exists a control $v(t) \in L^{2}(0, T)$ such that the unique corresponding mild solution of the system (36), which is defined to be

$$
Z(t)=e^{A_{0} t} Z_{0}+\int_{0}^{t} e^{A_{0}(t-s)} b v(s) d x
$$

satisfies $Z(T)=Z_{1}$. System (36) is called approximately controllable on $[0, T]$ if for any given states $Z_{0}, Z_{1} \in H$ and constant $\epsilon>0$, there exists a control $v(t) \in L^{2}(0, T)$ such that $\left\|Z(T)-Z_{1}\right\|<\epsilon$.

Since $A_{0}$ generates a $C_{0}$-group, exact controllability is equivalent to what so called "null" exact controllability: that is, we may choose $Z_{1}=0$ in the Definition 1.

Proposition 1 System (36) is approximately controllable on $[0, T]$ for any $T>0$ in $H$ but never exactly controllable in $H$ for any $T>0$.

Proof Since the control operator $B_{0}$ defined by $B_{0} u=u b$ for any $u \in \mathbb{C}$ is compact from the control space $\mathbb{C}$ to $H$, system (36) is not 
exactly controllable (Theorem 4.1 .5 of [19]). For the approximate controllability, we notice that each eigenvalue of $A_{0}$ is algebraically simple and, it can be easily shown that each eigenfunction $\tilde{\Psi}_{n}=$ $\left(g_{n}, \mu_{n} g_{n}, I_{m} \mu_{n} g_{n}^{\prime}(0), M \mu_{n} g_{n}(1), J \mu_{n} g_{n}^{\prime}(1)\right)$ of $A_{0}$ satisfies

$$
\left\langle b, \tilde{\Phi}_{n}\right\rangle_{H \times H}=I_{m} \mu_{n} g_{n}^{\prime}(0) \neq 0 .
$$

It follows from Proposition 3.13 at p. 61 of [10] that system (36) is approximately controllable.

However, our next result shows that the system (36) is exactly controllable when it is confined to the space $H_{1}=\left[D\left(A_{0}\right)\right]$, the graph space of $A_{0}$. Let us show the process. Consider $H_{1}$ to be the pivot space and let $H_{2}=\left[D\left(A_{0}^{2}\right)\right]$. Then $H_{2} \subset H_{1} \subset H_{2}^{\prime}$, where $H_{2}^{\prime}$ denotes the dual space of $H_{2}$. Hence $b \in H \subset H_{2}^{\prime}$. By definition, for any $Z=\left(f, g, \mathrm{I}_{m} g^{\prime}(1), M g(1), J g^{\prime}(1)\right) \in H_{2}$,

$$
\langle b, Z\rangle_{H_{2}^{\prime} \times H_{2}}=\left\langle b, A_{0}^{*} A_{0} Z\right\rangle_{H \times H}=-g^{\prime \prime}(0)+\alpha g^{\prime}(0) .
$$

Note that $A_{0}$ generates a $C_{0}$-semigroup (still denoted by $e^{A_{0} t}$ ) in $H_{1}$ with domain $\mathrm{H}_{2}$. Recall that $b$ is admissible (see e.g. [11]) with respect to $e^{A_{0} t}$ on $H_{1}$ if

$$
\left\langle b, e^{A_{0}^{*} t} Z\right\rangle_{H_{2}^{\prime} \times H_{2}}
$$

can be extended to be a continuous mapping from $H_{1}$ to $L^{2}(0, T)$ for some $T>0$.

Proposition $2 b$ is admissible for the semigroup $e^{A_{0} t}$ on $H_{1}$.

Proof For any $Z=\sum_{n \in \mathbb{Z}} a_{n} \tilde{\Phi}_{n} \in H_{2}$,

$$
\left\langle b, e^{A_{0}^{*} t} Z\right\rangle_{H_{2}^{\prime} \times H_{2}}=\sum_{n \in \mathbb{Z}} a_{n} e^{-\mu_{n} t}\left[-\mu_{n} g_{n}^{\prime \prime}(0)+\alpha \mu_{n} g_{n}^{\prime}(0)\right] .
$$

Since all $\mu_{n}$ are separated by a positive distance, it follows from a classical result due to Ingham [12] that for any $T>0$ there exists a constant $D_{T}>0$ such that

$$
\int_{0}^{T}\left|\left\langle b, e^{A_{0}^{*} t} Z\right\rangle_{H_{2} \times H_{2}^{\prime}}\right|^{2} d t \leq D_{T} \sum_{n \in \mathbb{Z}}\left|a_{n}\left[-\mu_{n} g_{n}^{\prime \prime}(0)+\alpha \mu_{n} g_{n}^{\prime}(0)\right]\right|^{2} .
$$


However, it follows from (26) and (29) that

$$
\left|-\mu_{n} g_{n}^{\prime \prime}(0)+\alpha \mu_{n} g_{n}^{\prime}(0)\right|=2\left|\mu_{n}\right|\left[1+\mathcal{O}\left(n^{-1}\right)\right] .
$$

Hence

$$
\int_{0}^{T}\left|\left\langle b, e^{A_{0}^{*} t} Z\right\rangle_{H_{1}^{\prime} \times H_{2}}\right|^{2} d t \leq \tilde{C}_{T} \sum_{n \in \mathbb{Z}}\left|a_{n} \mu_{n}\right|^{2} \leq C_{T}\|Z\|_{H_{1}}^{2}
$$

for some $\tilde{C}_{T}, C_{T}>0$, proving the result.

By Proposition 2, system (36) admits a unique solution in $H_{1}$ for each $Z(0)=Z_{0} \in H_{1}$, which can be expressed as

$$
Z(t)=e^{A_{0} t} Z_{0}+B(t) v
$$

where (see [11]) $B(t)$ is a strongly continuous family of bounded operators $B(t): L^{2}(0, T) \rightarrow H_{1}$ extended by

$$
\langle B(t) v, Z\rangle_{H_{1}^{\prime} \times H_{1}}=\int_{0}^{t}\left\langle b, e^{A_{0}^{*}(t-s)} Z\right\rangle_{H_{2}^{\prime} \times H_{2}} v(s) d s, \quad \forall Z \in H_{2} .
$$

THEOREM 4 There exists a $T_{0}>0$ such that for any $T>T_{0}$, system (36) is exactly controllable on $[0, T]$ in $H_{1}$.

Proof By duality principle (see $[13]),\left(A_{0}, b\right)$ is exact controllable $[0, T]$ if and only if $\left(A_{0}^{*}, b^{*}\right)=\left(-A_{0}, b^{*}\right)$ is exact observable on $[0, T]$, where $b^{*}$ is the adjoint operator of $b$ which is considered to be an operator from input space $\mathbb{C}$ to $H_{2}^{\prime}$. Precisely, there exists a constant $T_{0}>0$ such that for each $T>T_{0}$, there exists a constant $C_{T}>0$ such that

$$
\int_{0}^{T}\left|b^{*} e^{A_{0}^{*} t} Z\right|^{2} d t \geq C_{T}\|Z\|_{H_{1}}^{2} \quad \text { for any } z \in H_{1}
$$

Since $b$ is admissible, by duality, $b^{*}$ is an admissible observation element ([11]), that is $b^{*} e^{A_{0}^{*} t} Z$ is well-defined for any $T>0$ in the sense that $Z \rightarrow b^{*} e^{A_{0}^{*} t} Z$ is a continuous mapping from $H_{1}$ to $L^{2}(0, T)$. 
Suppose $Z=\sum_{n \in \mathbb{Z}} a_{n} \tilde{\Phi}_{n} \in H_{1}$. Then

$$
b^{*} e^{A_{0}^{*} t} Z=\sum_{n \in \mathbb{Z}} a_{n} e^{-\mu_{n} t}\left[-\mu_{n} g_{n}^{\prime \prime}(0)+\alpha \mu_{n} g_{n}^{\prime}(0)\right] .
$$

Again by (39) and Ingham Theorem, there exists an $T_{0}>0$ such that for any $T>T_{0}$, there are constants $\tilde{C}_{T}, C(T), C_{T}>0$ such that

$$
\begin{aligned}
\int_{0}^{T}\left|b^{*} e^{A_{0}^{*} t} Z\right|^{2} d t & \geq \tilde{C}_{T} \sum_{n \in \mathbb{Z}}\left|a_{n}\left[-\mu_{n} g_{n}^{\prime \prime}(0)+\alpha \mu_{n} g_{n}^{\prime}(0)\right]\right|^{2} \\
& \geq C(T) \sum_{n \in \mathbb{Z}}\left|a_{n} \mu_{n}\right|^{2} \geq C_{T}\|Z\|_{H_{1}}^{2} .
\end{aligned}
$$

The proof is complete.

Theorem 4 may imply the exponential stability of the closed-loop of the following collocated output feedback system in $\mathrm{H}_{1}$ :

$$
\left\{\begin{array}{l}
\frac{d}{d t} Z(t)=\mathcal{A}_{0} Z(t)+b v(t), \quad b=(0,0,1,0,0) \in H, \\
O(t)=b^{*} Z(t), \\
v(t)=k O(t) .
\end{array}\right.
$$

For general theory, we refer to [20]. Actually, for any $k>0$, let $\beta=k \alpha$. If $Z_{0}=\left(y_{0}, y_{1}, I_{m} y_{1}^{\prime}(0), M y_{1}(1), J y_{1}^{\prime}(1)\right) \in D\left(A_{0}\right)$, then $Y_{0}=$ $\left(y_{0}, y_{1}, I_{m} y_{1}^{\prime}(0)-k y_{0}^{\prime \prime}(0), M y_{1}(1), J y_{1}^{\prime}(1)\right) \in D(A)$. Let $\quad Y(t)=(y(\cdot, t)$, $\left.y_{t}(\cdot, t), I_{m} y_{x t}(0, t)-k y_{x x}(0, t), M y_{t}(1, t), J y_{x t}(1, t)\right)$ be the solution to $(6)$ with $\quad Y(0)=Y_{0} . \quad$ Then $Z(t)=\left(y(\cdot, t), y_{t}(\cdot, t), I_{m} y_{x t}(0, t), M y_{t}(1, t)\right.$, $\left.J y_{x t}(1, t)\right)$ satisfies (44) with $Z(0)=Z_{0}$ in the strong sense of $H$ norm. Since $e^{A t}$ is exponentially stable on $H$ and $Y_{0} \in D(A)$, we have

$$
\left\|A e^{A t} Y_{0}\right\|_{H}=\left\|e^{A t} A Y_{0}\right\|_{H} \leq\left\|e^{A t}\right\|\left\|A Y_{0}\right\|_{H} \leq M e^{-\omega t}\left\|A Y_{0}\right\|_{H}
$$

for some $\mathbf{M}, \omega>0$. However,

$$
\begin{aligned}
\left\|A e^{A t} Y_{0}\right\|_{H}^{2}= & \int_{0}^{1}\left[y_{x x x x}^{2}(x, t)+\left[y_{x x t}^{2}(x, t)\right] d x+\alpha\left|y_{x t}(0, t)\right|^{2}+I_{m}^{-1} \mid y_{x x}(0, t)\right. \\
& -\alpha y_{x}(0, t)-\left.\beta y_{x t}(0, t)\right|^{2}+M^{-1}\left|y_{x x x}(1, t)\right|^{2}+J^{-1}\left|y_{x x}(1, t)\right|^{2}
\end{aligned}
$$




$$
\begin{aligned}
\|Z(t)\|_{H_{1}}^{2}= & \int_{0}^{1}\left[y_{x x x x}^{2}(x, t)+y_{x x t}^{2}(x, t)\right] d x+\alpha\left|y_{x t}(0, t)\right|^{2}+I_{m}^{-1} \mid y_{x x}(0, t) \\
& -\left.\alpha y_{x}(0, t)\right|^{2}+M^{-1}\left|y_{x x x}(1, t)\right|^{2}+J^{-1}\left|y_{x x}(1, t)\right|^{2}
\end{aligned}
$$

Hence there are constants $C_{1}, C_{2}>0$ such that

$$
C_{1}\left\|A e^{A t} Y_{0}\right\|_{H} \leq\|Z(t)\|_{H_{1}} \leq C_{2}\left\|A e^{A t} Y_{0}\right\|_{H}
$$

Therefore,

$$
\|Z(t)\|_{H_{1}} \leq C_{2} / C_{1} M e^{-\omega t}\left\|Z_{0}\right\|_{H_{1}} .
$$

That is, the closed-loop of the system (44) is exponentially stable in $H_{1}$.

Finally, we relate the system (34) to an optimal control problem.

For any $T>0$, let

$$
\begin{aligned}
E(T)= & \frac{1}{2} \int_{0}^{1}\left[y_{x x}^{2}(x, t)+y_{t}^{2}(x, t)\right] d x+\frac{\alpha}{2} y_{x}^{2}(0, t) \\
& +\frac{I_{m}}{2} y_{x t}^{2}(0, t)+\frac{M}{2} y_{t}^{2}(1, t)+\frac{J}{2} y_{x t}^{2}(1, t)
\end{aligned}
$$

be the total energy of the system (34). Set

$$
J(v)=E(T)+\frac{\beta}{2} \int_{0}^{T} y_{x t}^{2}(0, t) d t+\frac{1}{2 \beta} v^{2}(t) d t .
$$

Since $E(T)=E(0)+\int_{0}^{T} y_{x t}(0, t) v(t) d t$, a straightforward calculation shows that (see also [21])

$$
\begin{aligned}
J(v) & =E(0)+\frac{1}{2} \int_{0}^{T} \beta^{-1}\left[v(t)+\beta y_{x t}(0, t)\right]^{2} d t \geq E(0) \\
J\left(v^{*}\right) & =E(0), \quad v^{*}(t)=-\beta y_{x t}(0, t),
\end{aligned}
$$

That is $v^{*}(t)=-\beta y_{x t}(0, t)$ is the optimal feedback control of system (34) under cost functional (47). But asymptotic expression (17) shows that system (34) is never exponentially stable under this optimal feedback control. Our result of present article shows that in order to 
uniformly stabilize system (34), we need a "high derivative" feedback control $v(t)=-\beta y_{x t}(0, t)+k y_{x x t}(0, t)$. The related optimal control problem needs further investigations.

\section{NUMERICAL SIMULATION}

Due to the spectrum-determined growth condition claimed by Theorem 2, the growth order $\omega(A)$ of the system (6), that is,

$$
\omega(A)=\inf \left\{\omega \mid E(t) \leq M e^{\omega t} E(0), \quad \text { for some } M \geq 1\right\}
$$

where $E(t)$ is defined by (46), is identical to the supremum of real parts of those $\lambda$ satisfying (14) with some nonzero $\phi$, which is a finite dimensional problem. It is seen from (17) that if $k=0$, then $\omega(A)=0$. When $k>0$, Theorem 3 tells us that $\omega(A)<0$ provided that $\beta \geq k \alpha$. For given system parameters $M, J, I_{m}, \alpha$, denote specifically by $\omega_{\beta, k}(A)$ the growth order $\omega(A)$ with feedback gains $\beta$ and $k$. One of optimization problems is to find optimal $\left(\beta^{*}, k^{*}\right)$ such that $\omega_{\beta^{*}, k^{*}}(A) \leq \omega_{\beta, k}(A)$ for any $\beta>0, k>0$. In this section, we shall use spectral method to calculate $\omega_{\beta, k}(A)$ [22]. Starting from eigenvalue problem (14) and setting

$$
f(x)=\phi\left(\frac{x+1}{2}\right)
$$

we obtain

$$
\left\{\begin{array}{l}
f^{\prime \prime \prime \prime}(x)+\lambda^{2} f(x)=0, \quad-1<x<1, \\
f(-1)=\left(I_{m} \lambda^{2}+\beta \lambda+\alpha\right) f^{\prime}(-1)-2(k \lambda+1) f^{\prime \prime}(-1)=0, \\
8 f^{\prime \prime \prime}(1)-M \lambda^{2} f(1)=0, \\
2 f^{\prime \prime}(1)+J \lambda^{2} f^{\prime}(1)=0 .
\end{array}\right.
$$

Let $P_{n}(x)$ be the Legendre polynomial of degree $n$, satisfying

$$
\left\{\begin{array}{l}
\frac{d}{d x}\left[\left(1-x^{2}\right) \frac{d}{d x} P_{n}(x)\right]+n(n+1) P_{n}(x)=0, \\
P_{n}(1)=1 .
\end{array}\right.
$$


We approximate $f(x)$ by $f_{N}(x)$

$$
f_{N}(x)=\sum_{n=0}^{N} a_{n} P_{n}(x) .
$$

We refer the procedure to [22] for details. Here we take $N=100$. Using this method, the total of 101 eigenvalues on the up half complex plane are easily calculated by MATLAB in PC. As it was indicated in [22] that in computing eigenvalues of boundary value problems with any discretization method, only those numerical values of small magnitude have significant accuracy, we only conclude the first 50 eigenvalues on the up half complex plane although, for our problem, there is no big change even for large magnitude eigenvalues.

Here we take $I_{m}=M=J=\alpha=1$. Figure 1 shows the functional relation of $\omega_{\beta, 1}(A)$ with respect to $\beta$ for $\beta=0$ to $\beta=6$. Figure 2 demonstrates the same relation of $\omega_{1, k}$ with respect to $k$. Both cases suggest us that the optimal $\left(\beta^{*}, 1\right)$ and $\left(1, k^{*}\right)$ do exist. An interesting fact is that our assumption $\beta \geq k \alpha$ may be necessary since from these two pictures that $\omega(A)$ can be positive outside region $\beta \geq k \alpha$.

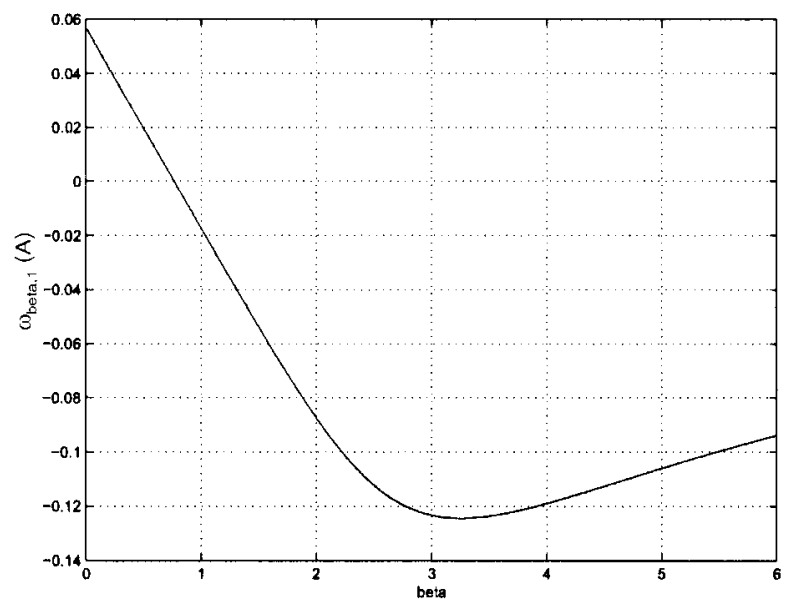

FIGURE 1 Functional relation between $\omega_{\beta, 1}(A)$ and $\beta\left(I_{m}=J=M=\alpha=1\right)$. 


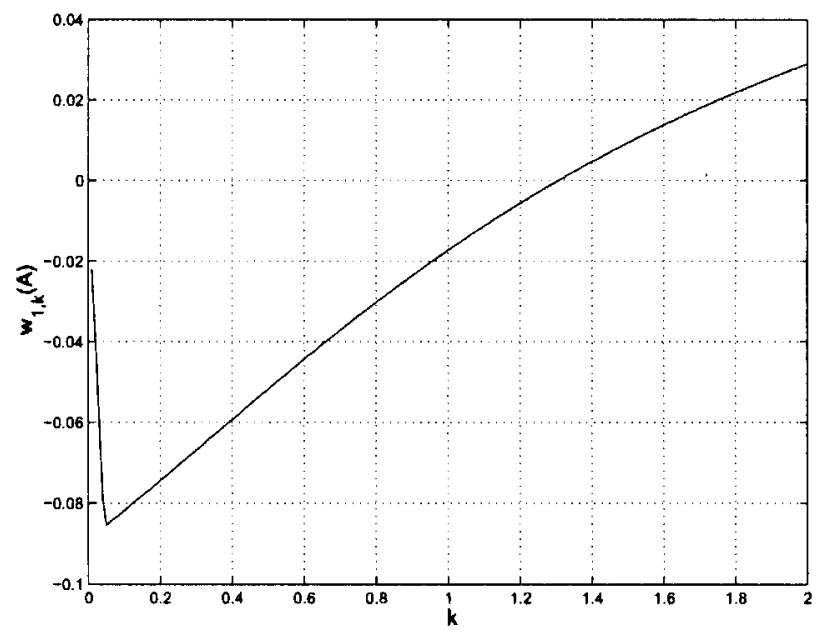

FIGURE 2 Functional relation between $\omega_{1, k}(A)$ and $k\left(I_{m}=J=M=\alpha=1\right)$.

\section{CONCLUDING REMARKS}

In this article, we consider the tracking control problem of a flexible beam with a tip rigid body. A "high derivative" linear feedback control is designed regardless of dissipativity of the system. By use of an abstract result on basis generation of discrete operators in Hilbert spaces, we show that the closed-loop system is a Riesz spectral system [19]: there is a sequence of the generalized eigenfunctions of the system, which forms a Riesz basis for the state Hilbert space. In the process of verification of the basis property, an asymptotic expression of eigenpairs is easily obtained. As a consequence, the exponential stability, the controllability of the system are concluded. Comparing with [16], our dynamic equation is more complicated: it considers not only the mass and moment of inertia of the tip rigid body, but also the counterparts of driven motor. This presents the states of system 5 components. Moreover, unlike the system in [16], because of lacking dissipativity, some well-known methods such as multiplier method can not be used to obtain the exponential stability (even if well-posedness) of the system studied in present article. 


\title{
Acknowledgment
}

\author{
The support of the National Natural Science Foundation of China is \\ gratefully acknowledged.
}

\section{References}

[1] B.Z. Guo and Q. Song (1995). Tracking control of a flexible beam by nonlinear boundary feedback. Journal of Applied Mathematics and Stochastic Analysis, 8, $47-58$.

[2] B.P. Rao (1993). Decay estimates of solution for a hybrid system of flexible structures. Eur. J. Appl. Math., 4, 303-319.

[3] W. Littman and L. Markus (1988). Exact boundary controllability of a hybrid system of elasticity. Arch. Rational Mech. Anal., 103, 193-236.

[4] Y. Sakawa and Z.H. Luo (1989). Modeling and control of coupled bending and torsional vibrations of flexible beams. IEEE Trans. on Auto. Control, 34, 970-977.

[5] B.P. Rao (1995). Uniform stabilization of a hybrid system of elasticity. SIAM J. Control and Optim., 33, 440-454.

[6] F. Conrad and O. Morgul (1998). On the stabilization of a flexible beam with a tip mass. SIAM J. Control and Optim., 36, 1962-1986.

[7] Z.H. Luo and B.Z. Guo (1997). Shear Force feedback control of a single link flexible robot with revolute joint. IEEE Trans. on Automatic Control, 42, 53-65.

[8] B.Z. Guo and R. Yu (2001). The Riesz basis property of discrete operators and application to a Euler-Bernoulli beam equation with boundary linear feedback control. IMA J. of Math. Control and information, 18, 241-251.

[9] Z.H. Luo (1993). Direct strain feedback control of flexible robot arms: new theoretical and experimental result. IEEE Trans. on Automatic Control, 38, 1610-1621.

[10] R.F. Curtain and J.A. Prichart (1978). Infinite dimensional linear systems theory. Lect. Note in Control and Information Sciences, 9.

[11] L.F. Ho and D.L. Russell (1983). Admissible input elements for systems in Hilbert space and a Carleson measure criterion. SIAM J. Control and Optim., 21, 615-640.

[12] A.E. Ingham (1936). Some trigonometrical inequalities with applications to the theory of series. Mathematische Zeitschrift, 41, 367-379.

[13] D. Russell and G. Weiss (1994). A general necessary condition for exact observability. SIAM J. Control and Optim., 32, 1-23.

[14] O. Morgul (1991). Orientation and stabilization of a flexible beam attached to a rigid body: planar motion. IEEE Trans. on Automatic Control, 36, 953-962.

[15] O. Morgul (1990). Control and stabilization of a flexible beam attached to a rigid body. Int. J. Control, 51, 11-33.

[16] B.Z. Guo (2001). Riesz basis approach to the stabilization of a flexible beam with a tip mass. SIAM J. Control and Optim., 39, 1736-1747.

[17] A. Pazy (1983). Semigroups of Linear Operators and Applications to Partial Differential Equations. Springer-Verlag, New York.

[18] S. Lang (1993). Complex Analysis. Springer-Verlag, New York.

[19] R.F. Curtain and H.J. Zwart (1995). An Introduction to Infinite Dimensional Linear Systems Theory. Springer-Verlag, New York.

[20] B.Z. Guo and Y.H. Luo (2002). Controllability and stability of a second order hyperbolic system with collocated sensor/actuator. Systems and Control Letters, 46, 45-65. 
[21] J. Leblond and J.P. Marmorat (1987). Stabilization of a vibrating beam: a regularity result. Proceeding of the Workshop on Stabilization of Flexible Structures, pp. 162183. COMCON, Optimization Software Inc.

[22] G. Chen, S.G. Krantz, D.L. Russell, C.E. Wayne, H.H. West and M.P. Coleman (1989). Analysis, design, and behavior of dissipative joints for coupled beams. SIAM on Applied Mathematics, 49, 1665-1989. 
Copyright of Optimization Methods \& Software is the property of Taylor \& Francis Ltd and its content may not be copied or emailed to multiple sites or posted to a listserv without the copyright holder's express written permission. However, users may print, download, or email articles for individual use. 